\title{
Very Important Person Syndrome: A Psychosocial Problem!
}

\author{
Anshuman Darbari
}

Received: 10 September 2011 / Accepted: 6 March 2012 / Published online: 16 March 2012

(C) Association of Surgeons of India 2012

\section{Dear Sir,}

All of us whether physicians or surgeons related to any medical field often get the privilege of treating very important persons (VIP). The VIP patients may be socially, politically, administratively, or even medically affluent persons. Being in the medical profession, one can't discriminate people on grounds of sex, religion, income, or social group. But the problem arises when the patients or their caretakers start searching for the best available treatment, and in pursuit of the same they sometimes, knowingly or unknowingly, breach the trust of their physicians. Thus, this 'pick and choose' approach of the patient towards a physician creates a fracture in relation [1].

The deficit of trust also evolves when the physician treating such VIP persons receives multiple opinions about ailment and suggested line of treatment from those medical experts who are neither involved in the management of the patient at any stage nor have any idea about the ground realities. Doctors in big cities or in a multispecialty hospital generally offer such opinions just because the patient in question is an influential one, which does not seem to be justifiable [2].

In the midst of a variety of medical opinions and prevailing pressure of treating a VIP patient, the physician lands up in an environment that is very vulnerable and may jeopardize the medical management. If not addressed properly, this problem may also invite conflicts, litigations, and violent episodes as patients disagree with recommendations that the primary physician believes are in the patient's best interest. This problem is mainly being faced in rural and semi-urban regions. Sometimes, this situation may arise due to a lack of interpersonal communication among the patients, attendants, their physicians, and members of the medical community, so it can all lead to strong feeling of disagreement [3].

I'm throwing this thought and raising the topic because this is an issue of significant psychological value for both the patients as well as the doctors and entails detailed deliberation. It's a matter of debate that most of these types of patients lend in complication due to over enthusiastic treatment or get proper judicious management!

\section{References}

1. MCI-Rules and Regulations/Code of Medical Ethics Regulations 2002

2. Desai P (1988) Medical ethics in India. J Med Philos 3:231-255

3. http://en.wikipedia.org/wiki/Medical_ethics 